\title{
Composição química e toxicidade do óleo essencial de eucalipto sobre o ácaro-rajado
}

\author{
Lauana Pellanda de Souza*1, Hugo Bolsoni Zago², Patrícia Fontes Pinheiro², \\ Wilson Rodrigues Valbon', Vitor Zuim', Dirceu Pratissoli
}

'Universidade Federal de Viçosa, Viçosa, MG, Brasil

${ }^{2}$ Universidade Federal do Espírito Santo, Alegre, ES, Brasil

*Autor correspondente, e-mail: lauanaps@hotmail.com

\begin{abstract}
Resumo
O ácaro-rajado, Tetranychus urticae Koch, 1836 (Acari: Tetranychidae) é uma praga polífaga e causa grandes prejuízo a agricultura brasileira. O controle dessa praga geralmente é realizado por produtos de origem sintética. Com tudo, os óleos essenciais com propriedade acaricida, podem ser considerados uma alternativa no controle desta praga. Assim, os objetivos deste trabalho foram identificar a composição química e avaliar a toxicidade por fumigação do óleo essencial de Eucalyptus citriodora (Hook.) KD Hill \& LAS Johnson sobre T. urticae. A extração do óleo essencial foi realizada por hidrodestilação, utilizando-se equipamento do tipo Clevenger. A identificação dos compostos foi feita pela comparação dos espectros de massas obtidos com os padrões disponíveis no banco de dados da espectroteca Willey 330.000 e pelo índice de Kovats (IK) calculado para cada componente. Fêmeas adultas do ácaro-rajado foram submetidas às doses de 3,57; 7, 14; 10,$71 ; 14,28$ e 17,85 $\mu \mathrm{L} \mathrm{L}^{-1}$ de ar do óleo essencial em câmaras de fumigação por 24, 48 e $72 \mathrm{~h}$. Posteriormente, a concentração letal $\left(\mathrm{CL}_{50}\right)$ do óleo essencial foi estimada. Foram identificados dez compostos, sendo o citronelal $(68,20 \%)$ o composto presente em maior quantidade. $A_{s} C L_{50}$ encontradas após 24, 48 e 72 h de exposição ao óleo essencial foram, respectivamente, de 17,55, 17,00 e 10,50 $\mathrm{L} \mathrm{L} \mathrm{L}^{-1}$ de ar e a fecundidade das fêmeas de T. urticae sofreu reduções com o aumento das concentrações utilizadas.
\end{abstract}

Palavras-chave: Eucalyptus citriodora, fumigação, Tetranychus urticae

\section{Chemical composition and toxicity of eucalyptus essential oil on spotted spider mite}

\begin{abstract}
The spotted spider mite, Tetranychus urticae Koch, 1836 (Acari: Tetranychidae) is a polyphagous pest which causes extensive damage to Brazilian agriculture. The control of this pest is usually performed by synthetic origin products. Thus, the essential oils with acaricide property can be resources considered as an alternative to control this pest. The objective of this study was to identify the chemical composition and evaluate the fumigation toxicity of Eucalyptus citriodora (Hook.) KD Hill \& LAS Johnson essential oil on T. urticae. The essential oil extraction was performed by hydrodistillation, usinga Clevenger type apparatus. The compounds identification was made comparing the obtained mass spectra with the available spectra in the database of the spectral library Willey 330,000 and by the Kovats index (IK), calculated for each compound. Adult female spotted spider mite were subjected to doses of 3.57, 7.14, 10.71, 14.28 and $17.85 \mu \mathrm{L} \mathrm{L}^{-1}$ of the essential oil air in fumigation chambers during 24,48 and 72 hours. Subsequently, lethal concentration ( $\mathrm{LC}_{50}$ ) of the essential oil was estimated. Ten compounds were identified, being the citronellal (68.20\%) the major compound. The $\mathrm{LC}_{50}$ value observed after 24,48 and 72 hours of exposure to essential oil were 17.55, 17.00 and $10.50 \mu \mathrm{L} \mathrm{L}^{-1}$ of air, respectively, and the fecundity of $T$. urticae females reduced with an increase of the used concentrations.
\end{abstract}

Keywords: Eucalyptus citriodora, fumigation, Tetranychus urticae 


\section{Introdução}

O ácaro-rajado, Tetranychus urticae Koch, 1836 (Acari: Tetranychidae) está presente em grande parte do mundo e possui muitas plantas hospedeiras, aproximadamente 1100 espécies distribuídas em 70 gêneros (Grbic et al., 2011 1). Causa prejuízo em diversas culturas de valor econômico, principalmente em frutíferas, ornamentais e hortaliças (Moraes \& Flechtmann, 2008). Em especial no morangueiro, essa praga apresenta rápido desenvolvimento e alto potencial reprodutivo, com isso, atinge elevado nível de infestação (Nyoike \& Liburd, 2013).

Em decorrência do rápido prejuízo ocasionado pelo ácaro-rajado, geralmente adota-se o controle químico, e esse, apesar de eficiente, pode acarretar o surgimento de populações resistentes aos acaricidas, elevados índices de resíduos nos produtos alimentícios, contaminações ambientais e impactos negativos à saúde humana (Grützmacher et al., 2008; Peixoto et al., 2009; Sato et al., 2009; Ferreira et al., 2015). Mediante a esses problemas, pesquisas estão sendo realizadas com objetivo de empregar produtos de origem botânica no manejo de pragas (Ootani et al., 201 1; Roh et al., 2013; Zandi-Sohani \& Ramezani, 2015; Souza et al., 2015).

Diversas plantas vêm sendo estudadas como potenciais ferramentas no controle de insetos e ácaros-praga, e os óleos essenciais de algumas dessas plantas possuem comprovada atividade acaricida (Choi et al., 2004; OliveroVerbel et al., 2010; Motazedian et al., 2012). Dentre os benefícios da utilização dos óleos essenciais no controle de pragas está o fato de alguns desses óleos oferecem menores risco ao homem e maior biodegradabilidade no ambiente quando comparados aos produtos sintéticos (Dayan et al., 2009).

Dentre as plantas com potencial para produção de óleos essenciais com atividade praguicida, merecem destaque as espécies da família Myrtaceae, em especial as do gênero Eucalyptus, que além de apresentarem boa adaptação as condições climáticas do Brasil, possuem também elevada produção de óleo essencial (Estanislau et al., 2001; Castro et al., 2008).
Várias propriedades biológicas já foram atribuídas ao gênero Eucalyptus, entre elas atividade inseticida contra besouros (Brito et al., 2006), ação repelente contra Phlebotomus papatasi (Yaghoobi-Ershadi et al., 2006), além de repelência e atividade acaricida contra $T$. urticae (Lim et al., 2012; Roh et al., 2013). No Brasil, as principais espécies de Eucalyptus usadas para a produção de óleos essenciais comerciais são E. staigeriana, E. citriodora e E. globulus (Vitti \& Brito, 2003).

Mediante ao exposto, o objetivo deste trabalho foi identificar a composição química e avaliar a toxicidade por fumigação do óleo essencial de Eucalyptus citriodora sobre Tetranychus urticae.

\section{Material e Métodos}

A extração do óleo essencial e os bioensaios foram realizados no setor de Entomologia do Núcleo de Desenvolvimento Científico e Tecnológico em Manejo Fitossanitário (NUDEMAFI) no Centro de Ciências Agrárias da Universidade Federal do Espírito Santo (CCAUFES).

Obtenção e multiplicação de Tetranychus urticae

A população de T. urticae utilizada nos bioensaios foi estabelecida a partir de coletas em plantios de morango no município de

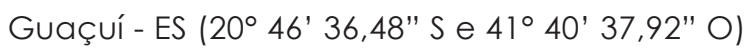
e a criação foi mantida a $25 \pm 1{ }^{\circ} \mathrm{C}$, U.R. $70 \pm 10 \%$ e fotofase de $12 \mathrm{~h}$, em plantas de feijão-deporco (Canavalia ensiformes L. DC, Fabaceae) que eram substituídas a cada cinco dias.

Obtenção e identificação dos compostos existentes no óleo essencial

Folhas frescas de Eucalyptus citriodora foram coletadas no período matutino, no município de Alegre, Sul do Espírito Santo, Brasil, entre os meses de março e novembro de 2012.

A extração do óleo essencial foi realizada por hidrodestilação, utilizando-se equipamento do tipo Clevenger. Em um balão de fundo redondo (3 L) foram adicionados $100 \mathrm{~g}$ de planta fresca, triturada manualmente, e 1,5 L de água destilada. Após destilação por 3 horas 
foi recolhido $100 \mathrm{~mL}$ de hidrolato (água + óleo). O óleo foi então extraído da fase aquosa com funil de separação, utilizando pentano como solvente. Foram realizadas três extrações com $30 \mathrm{~mL}$ de solvente cada e então adicionado sulfato de sódio anidro à fase orgânica, com o objetivo de remover a água presente. A fase orgânica foi filtrada e o solvente removido em evaporador rotativo. O óleo essencial foi acondicionado em frasco escuro envolto por papel laminado e armazenado em refrigerador a $4^{\circ} \mathrm{C}$, para que mantivesse as características originais até a análise e utilização no bioensaio. O rendimento do óleo essencial foi calculado dividindo-se a massa do material fresco pelo volume de óleo extraído.

O óleo essencial foi analisado por cromatografia em fase gasosa (CG) em equipamento Shimadzu GC-2010 Plus, equipado com detector de ionização de chama (DIC) de hidrogênio e coluna capilar Rtx-5MS, $30 \mathrm{~m}$ de comprimento e $0,25 \mathrm{~mm}$ de diâmetro interno. $\bigcirc$ gás de arraste utilizado foi $\bigcirc$ nitrogênio. A temperatura inicial da coluna foi de $60^{\circ} \mathrm{C}$, sendo programada para ter acréscimos de $3^{\circ} \mathrm{C}$ a cada minuto, até atingir a temperatura máxima de $240^{\circ} \mathrm{C}$. As temperaturas do injetor e do detector foram fixadas em 240 e $250^{\circ} \mathrm{C}$, respectivamente. Uma quantidade de $10 \mathrm{mg}$ do óleo foi diluída em $1 \mathrm{~mL}$ de diclorometano, sendo que $1 \mu \mathrm{L}$ dessa solução foi usado na injeção.

A identificação dos compostos do óleo essencial foi realizada por cromatografia gasosa acoplada ao espectrômetro de massas (CGEM), em equipamento com detector seletivo de massa, modelo QP-PLUS-2010 (ShimadzU). A coluna cromatográfica utilizada foi a capilar de silica fundida com fase estacionária Rtx-5MS, de $30 \mathrm{~m}$ de comprimento e $0,25 \mathrm{~mm}$ de diâmetro interno, utilizando hélio como gás de arraste. As temperaturas foram de $220^{\circ} \mathrm{C}$ no injetor e 300 ${ }^{\circ} \mathrm{C}$ no detector. A programação de temperatura no forno foi a mesma utilizada nas análises por CG-DIC.

A identificação dos compostos foi feita pela comparação dos espectros de massas obtidos com os disponíveis no banco de dados da espectroteca Willey 330.000 e pelo índice de
Kovats (IK) calculado para cada componente (Adams, 2007).

O percentual relativo de cada composto foi calculado através da razão entre a área integral de seus respectivos picos e a área total de todos os constituintes da amostra, dados estes obtidos pelas análises realizadas no cromatógrafo a gás com detector de ionização de chama.

Avaliação da atividade acaricida por fumigação

A metodologia para avaliar a ação fumigante do óleo essencial sobre T. urticae foi realizada segundo Souza et al. (2015). O teste de fumigação foi realizado submetendo fêmeas adultas do ácaro-rajado às doses de 3,$57 ; 7,14 ; 10,71 ; 14,28$ e $17,85 \mu \mathrm{L} \mathrm{L}^{-1}$ de ar, em câmaras de fumigação por 24, 48 e $72 \mathrm{~h}$. Nada foi aplicado no controle. Cada câmara de fumigação foi composta por um recipiente de vidro com volume de 1,4 L. No interior da câmara foram colocados três recipientes de vidro com volume de $10 \mathrm{~mL}$ contendo água, que serviam de suporte para um disco de folha de feijão-de-porco de $2,5 \mathrm{~cm}$ fixado pelo pecíolo com auxílio de algodão hidropônico. Para cada disco de feijão-de-porco foram transferidas dez fêmeas adultas de T. urticae.

$\mathrm{Na}$ borda superior da câmara de fumigação foi afixada uma fita de $2 \mathrm{~cm} \times 5$ $\mathrm{cm}$ de papel filtro onde foi depositado o óleo essencial com auxílio de pipetador automático. Após a aplicação do óleo, a parte superior da câmara de fumigação foi vedada com quatro camadas de plástico tipo PVC e as câmaras acondicionadas em ambiente controlado com temperatura de $25 \pm 1^{\circ} \mathrm{C}$, umidade relativa do ar de $70 \pm 10 \%$ e fotofase de $12 \mathrm{~h}$.

Para cada concentração e tempo de exposição ao óleo essencial foram realizadas nove repetições, sendo cada repetição composta por um disco de feijão-de-porco contendo dez fêmeas adultas de T. urticae.

As avaliações foram feitas ao final de 24 , 48 e $72 \mathrm{~h}$. O delineamento do experimento foi inteiramente casualisado (DIC) e as avaliações foram realizadas contando-se o número de ácaros mortos (incapazes de caminhar uma 
distância superior ao comprimento do corpo após um leve toque com pincel de cerdas finas, $n^{\circ} 000$ ). A fecundidade foi avaliada pela contagem do número de ovos presentes no disco de folha.

\section{Análises Estatísticas}

A $\mathrm{CL}_{50}$ foi estimada submetendose os dados de mortalidade a análise de Probit (Robertson et al., 2007). Os dados de fecundidade foram submetidos à análise de regressão, com auxílio do programa estatístico Assistat, versão 7.6 Beta.

\section{Resultados e Discussão}

Identificação dos compostos existentes no óleo essencial

O óleo essencial de Eucalyptus citriodora apresentou rendimento de $2,9 \%\left(\mathrm{~m} \mathrm{~m}^{-1}\right)$ da massa fresca e foram encontradas onze substâncias compondo o óleo analisado, porém uma das substâncias, que representa $8,69 \%$ da amostra, não pode ser identificada pela biblioteca do aparelho. O composto majoritário encontrado foi o citronelal, com $68,20 \%$, seguido do neoisopulegol, com 10,58\% (Tabela 1).

A composição química do óleo

Tabela 1. Composição química do óleo essencial de Eucalyptus citriodora

\begin{tabular}{ccccc}
\hline TR $(\mathbf{m i n})$ & Área (\%) & IK (Cal.) & IK (Tab.) & Composto Identificado \\
\hline 8,95 & 1,18 & 1037 & 1026 & Eucaliptol (1,8-cineol) \\
9,99 & 0,25 & 1061 & 1054 & $\gamma$-terpinene \\
11,62 & 0,36 & 1094 & 1095 & Linalol \\
13,54 & 10,58 & 1138 & 1144 & Neo-isopulegol \\
13,93 & 68,20 & 1146 & 1148 & Citronelal \\
14,57 & 0,60 & 1160 & 1155 & Iso-isopulegol \\
15,56 & 0,24 & 1179 & 1186 & $\alpha$-terpineol \\
17,19 & 8,69 & 1211 & - & N.I. ${ }^{*}$ \\
21,86 & 7,02 & 1307 & - & Acetato de $\alpha$-terpenila* \\
22,81 & 2,84 & 1329 & - & Acetato de citronelita* \\
24,61 & 0,04 & 1368 & - & Phosphoryl fluoride** \\
\hline TR= Tempo de Retenção em minutos; IK (cal.) = Indice de Kovats calculado; IK (tab.) = Indice de Kovats tabelado. \\
*Composto identificado somente pela biblioteca do aparelho. ' N.I. = composto não identificado.
\end{tabular}

essencial estudado corrobora com os resultados encontrado por Ootani et al. (2011), que observaram $61,78 \%$ de citronelal e 15,58\% de Isopulegol presentes no óleo essencial de E. citriodora. Também Han et al. (2011), analisando o óleo essencial da mesma espécie, encontraram $65,94 \%$ de citronelal.

O rendimento do óleo essencial estudado apresentou-se inferior ao encontrado por Estanislau et al. (2001), que observaram um rendimento de $4 \%$ de óleo essencial da mesma espécie em plantas cultivadas no estado de Goiás. Castro et al. (2008), estudaram - E. citriodora no estado de Minas Gerais e observaram que o rendimento do óleo essencial desta planta variou de 2,56 a $6,15 \%$ de acordo com o local e a época de coleta das plantas. Em estudo similar Chalchat et al. (2001), encontraram rendimentos de $1,0 \%$ a $2,6 \%$ de óleo essencial em plantas de E. citriodora cultivadas na Tailândia e de $0,8 \%$ a $1,3 \%$ em plantas cultivadas no Marrocos e em Israel.
De acordo com Castro et al. (2008), além do rendimento, a composição e a concentração de cada composto podem variar conforme - local e a época de coleta das plantas. Estes mesmos autores observaram uma redução no teor de citronelal de $10 \%$ de fevereiro para agosto e alguns compostos presentes no óleo oriundo de plantas coletadas em agosto, como o $\beta$-pineno $(0,58 \%)$, deixam de compor o óleo extraído de plantas coletadas em fevereiro na mesma localidade. Além do local e da época de colheita, a composição dos óleos essenciais pode sofrer influência de fatores como solo, clima e adubação. Carvalho et al. (2005), comparando o rendimento do óleo essencial de Cymbopogon citratus submetido a diferentes tipos de adubação, demonstraram que 0 tratamento com adubação orgânica gerou um rendimento do óleo superior ao tratamento com adubação convencional.

Os tempos de retenção das substâncias encontradas no óleo essencial estudado 
variaram de 8,95 a 24,61 minutos. Em testes de fumigação, onde não ocorre o contato direto do óleo essencial com a praga, deve-se dedicar uma atenção especial ao tempo de retenção dos compostos químicos encontradas em sua composição. O tempo de retenção está diretamente relacionado à velocidade que um composto demora a ser volatilizado no ambiente e, consequentemente, entrar em contato com a praga.

Atividade fumigante do óleo essencial sobre $T$. urticae

Os valores de inclinação das curvas de concentração-resposta $\left(\mathrm{CL}_{50}\right)$ calculados para o óleo essencial de E. citriodora sobre T. urticae estão descritos na Tabela 2, com os respectivos intervalos de confiança.

Tabela 2. Inclinação das curvas de concentração letal $\left(\mathrm{CL}_{50}\right)$ do óleo essencial de Eucalyptus citriodora sobre Tetranychus urticae.

\begin{tabular}{|c|c|c|c|c|c|}
\hline $\mathrm{T}^{\prime}(\mathrm{h})$ & $\mathbf{N}^{2}$ & Inclinação $\pm E^{3}{ }^{3}$ & 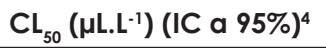 & $\mathrm{GL}^{5}$ & $X^{2(6)}$ \\
\hline 24 & 450 & $3,48 \pm 0,63$ & $17,55(15,94-20,51)$ & 3 & 0,51 \\
\hline 48 & 450 & $4,80 \pm 0,99$ & $17,00(15,23-20,20)$ & 3 & 0,10 \\
\hline 72 & 450 & $6,32 \pm 0,90$ & $10,50(7,41-12,24)$ & 3 & 5,18 \\
\hline
\end{tabular}
probabilidade; ${ }^{5}$ Número de graus de liberdade; ${ }^{6}$ Teste qui-quadrado.

Os valores de inclinação das curvas de concentração letal do óleo essencial de E. citriodora sobre T. urticae mostraramse diretamente proporcionais ao tempo de exposição ao óleo essencial. A inclinação da curva determina a variabilidade genética da população de insetos em relação a mortalidade provocada pelo óleo. Assim, menores valores de inclinação da reta indicam maior variabilidade genética, sugerindo a presença de mais de um genótipo na população testada, podendo resultar em maiores índices de resistência da população ao produto utilizado (Kerns \& Gaylor, 1992).

Foi observado redução na $\mathrm{CL}_{50}$ do óleo essencial de E. citriodora de 17,00 para $10,50 \mu \mathrm{L} \mathrm{L}^{-1}$ de ar, com o aumento do tempo de exposição ao óleo de 48 para 72 h. Essa redução na $\mathrm{CL}_{50}$ do óleo essencial é importante na sua possível utilização no manejo do ácarorajado, uma vez que a menor dose do óleo seria capaz de controlar a infestação com eficiência, no entanto, necessitaria de maior período de exposição da população ao óleo.

Em estudo com o óleo essencial de Eucalyptus globulus e E. citriodora, Choi et al. (2004) também observaram eleito desses óleos sobre a mortalidade de T. urticae. De acordo com os autores, E. citriodora apresentou maior toxicidade, sendo que 9,3 $\mathrm{L} \mathrm{L} \mathrm{L}^{-1}$ de ar do óleo foi capaz de controlar $99,0 \%$ da população da praga, enquanto que para E. globulus foram necessários 19,0 $\mu \mathrm{L} \mathrm{L}^{-1}$ de ar para controlar 89,0\% da população.

Investigações prévias em relação ao potencial inseticida e acaricida de óleos essenciais de diferentes plantas têm mostrado, em geral, que a toxicidade apresentada está relacionada aos componentes majoritários dos óleos essenciais, que no caso do $E$. citriodora é o monoterpeno citronelal $(68,20 \%)$, conhecidamente possuidor de atividades acaricidas (Han et al., 2011; Ootani et al., 2011). No entanto, não se devem descartar o possível efeito dos compostos presentes em menores quantidades no óleo essencial e até mesmo a ocorrência de efeito sinérgico entre compostos presentes em maior e menor quantidades. Estudos realizados por Han et al. (2011), demonstram que após 24 horas de exposição, o acetato de citronelol obteve $\mathrm{CL}_{50}$ de $16,8 \mu \mathrm{g} \mathrm{cm}$ e o citronelal de $33,9 \mu \mathrm{g} \mathrm{cm}^{-3}$, esses presentes no óleo essencial de E. citriodora em percentuais de 2,2 e 65,94\%, respectivamente. Assim, mesmo os compostos químicos presentes em menor quantidade nos óleos essenciais podem ser importantes fontes de estudo para a produção de acaricidas.

A fecundidade de T. urticae, obtida pelo número de ovos por fêmea, foi inversamente proporcional à concentração utilizada nos três tempos de exposição à fumigação (Figura 1).

No presente estudo, além de mortalidade, o óleo essencial de E. citriodora 


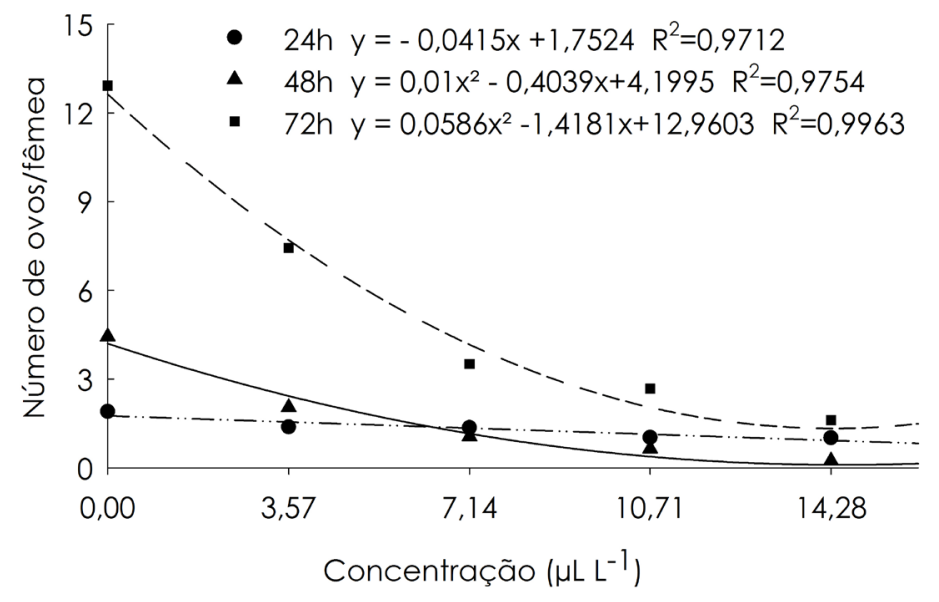

Figura 1. Fecundidade de T. urticae com 24, 48 e 72 horas de exposição ao óleo essencial de E. citriodora.

apresentou redução da fecundidade de $T$. urticae (Figura 1). O mesmo foi observado por Roh et al. (2013), em teste de imersão com óleos essenciais de Eucalyptus bicostata, E. maidenii, E. sideroxylon, E. approximans a $5 \%$ (5 $\mathrm{ml} \mathrm{L}^{-1}$ ) para o mesmo ácaro. Óleos essenciais de outras espécies de plantas também promovem redução da fecundidade de $T$. urticae. Folhas e frutos de Xylopia sericea (Annonaceae) e Protium heptaphyllum (Burceraceae), promoveram reduções na oviposição de $T$. urticae à medida que as concentrações do óleo foram aumentadas até $10 \mu \mathrm{L} \mathrm{L-1}$ de ar (Pontes et al., 2007a; Pontes et al. 2007b).

Os monoterpenos, como os encontrados no óleo essencial de E. citriodora, vêm sendo citados na literatura como responsável para inibição do crescimento, maturação prejudicada, reduzida capacidade de reprodução, supressão do apetite e morte de insetos (Viegas Júnior, 2003). Apesar do mecanismo de ação dos monoterpenos no organismo dos insetos e ácaros ser desconhecida, Coats et al. (1991) afirmam que a atividade praguicida se deve provavelmente a ação neurotóxica apresentada por estas substâncias.

Testes futuros devem ser realizados, principalmente a campo, no sentido de estudar - mecanismo de ação dos constituintes dos óleos essenciais e seu efeito sobre a cultura, o homem e o ambiente.

\section{Conclusões}

Foram encontradas onze substâncias compondo o óleo essencial de E. citriodora e o composto presente em maior quantidade é o citronelal $(68,20 \%)$.

Os resultados indicam que o óleo essencial de E. citriodora possui efeito acaricida, podendo ser usado via fumigação contra o ácaro-rajado e em pequenas doses reduz a fecundidade das fêmeas.

\section{Referências}

Adams, R.P. 2007. Identification of essention oil components by gas chromatography/mass spectroscopy. 4 ed. Allured Publishing Corp, Carol Stream, USA. 804p.

Brito, J.P., Oliveira, J.E.M., Bortoli, S.A. 2006. Toxicidade de óleos essenciais de Eucalyptus spp. sobre Callosobruchus maculatus (Fabr., 1775) (Coleoptera: Bruchidae). Revista de Biologia e Ciências da Terra 6: 96-103.

Carvalho, C.M., Costa, C.P.M., Sousa, J.S., Silva, R.H.D., Oliveira, C.L., Paixão, F.J.R. 2005. Rendimento da produção de óleo essencial de capim-santo submetido a diferentes tipos de adubação. Revista de Biologia e Ciências da Terra 5: 96-103.

Castro, N.E.A., Carvalho, M.G., Pimentel, F.A., Correa, R.M., Guimarães, L.G.L. 2008. Avaliação de rendimento e dos constituintes químicos do óleo essencial de folhas de Eucalyptus citriodora Hook colhidas em diferentes municípios de Minas Gerais. Revista Brasileira de Plantas Medicinais 10: 70-75. 
Chalchat, J.C., Kundakovic, T., Gorunovic, M.S. 2001. Essential oil the leaves of Eucalyptus camaldulensis Dehn, Myrtaceae from Jerusalem. Jornal of Essencial Oil Research 13: 105-107.

Choi, W.I., Lee, S.G., Park, H.M, Ahn, Y.J. 2004. Toxicity of Plant Essential Oils to Tetranychus urticae (Acari: Tetranychidae) and Phytoseiulus persimilis (Acari: Phytoseiidae). Journal of Economic Entomology 97: 553-558.

Coats, J.R., Karr, L.L., Drewes, C.D., 1991. Toxicity and neurotoxic elects of monoterpenoids in insects and earthworms. American Chemical Society Symposium Series 449: 306-316.

Dayan, F.E., Cantrell, C.L., Duke, S.O. 2009. Natural products in crop protection. Bioorganic \& Medicinal Chemistry 17: 4022-4034.

Estanislau, A.A., Barros, F.A.S., Peña, A.P., Santos, S.C., Ferri, P.H., Paula, J.R. 2001. Composição química e atividade antibacteriana dos óleos essenciais de cinco espécies de Eucalyptus cultivada em Goiás. Revista Brasileira de Farmacognosia 11: 95-100.

Ferreira, C.B.S., Andrade, F.H.N., Rodrigues, A.R.S., Siqueira, H.A.A., Gondim Júnior, M.G.C. 2015. Resistance in field populations of Tetranychus urticae to acaricides and characterization of the inheritance of abamectin resistance. Crop Protection 67: 77-83.

Grbic, M., van Leeuwen, T., Clark, R.M., Rombauts, S., Rouzé, P., Grbic, V., Osborne, E.J., Dermauw, W., Ngoc, P.C.T., Ortego, F., Crespo, P.H., Diaz, I., Martinez, M., Navajas, M., Sucena, É., Magalhães, S., Nagy, L., Pace, R.M., Djuranovic, S., Smagghe, G., Iga, M., Christiaens, O., Veenstra, J.A., Ewer, J., Villalobos, R.M., Hutter, J.L., Hudson, S.D., Velez, M., Yi, S.V., Zeng, J., da Silva, A.P., Roch, F., Cazaux, M., Navarro, M., Zhurov, V., Acevedo, G., Bjelica, A., Fawcett, J.A., Bonnet, E., Martens, C., Baele, G., Wissler, L., Rodriguez, A.S., Tirry, L., Blais, C., Demeestere, K., Henz, S.R., Gregory, T.R., Mathieu, J., Verdon, L., Farinelli, L., Schmutz, J., Lindquist, E.E., Feyereisen, R., de Pee, Y.V., 2011. The genome of Tetranychus urticae reveals herbivorous pest adaptations. Nature 479: 487492.

Grützmacher, D. D., Grützmacher, A. D., Agostinetto, D., Loeck, A.E., Roman, R., Peixoto, S.C., Zanella, R. 2008. Monitoramento de agrotóxicos em dois mananciais hídricos no sul do Brasil. Revista Brasileira de Engenharia Agrícola e Ambiental 12: 632-637.

Han, J., Kim, S., Choi, B.R., Lee, S.G., Ahn, Y.J. 2011. Fumigant toxicity of lemon eucalyptus oil constituents to acaricide-susceptible and acaricide-resistant Tetranychus urticae. Pest Management Science 67: 1583-1588.
Kerns, D.L., Gaylor, M.J. 1992. Insecticide resistance in field populations of the cotton aphid (Homoptera: Aphididae). Journal of Economic Entomology 85: 1-8.

Lim, E., Lee, B.H, Park, C.G. 2012. Fumigant activity of essential oils and their components from Eucalyptus codonocarpa and E. dives against Tetranychus urticae (Acari: Tetranychidae) at three temperatures. Journal of Applied Entomology. 136: 698-703.

Moraes, G.J., Flechtmann, C.H.W., 2008. Manual de acarologia: acarologia básica e ácaros de plantas cultivadas no Brasil. Editora Holos, Ribeirão Preto, Brasil. 308p.

Motazedian, N., Ravan, S., Bandani, A.R. 2012. Toxicity and Repellency Effects of Three Essential Oils against Tetranychus urticae Koch (Acari: Tetranychidae). Journal of Agricultural Science and Technology 14: 275-284.

Nyoike, T.W., Liburd, O.E. 2013. Effect of Tetranychus urticae (Acari: Tetranychidae), on marketable yields of field-grown strawberries in North-Central Florida. Journal of Economic Entomology 106: 1757-1766.

Olivero-Verbel, J., Nerio, L.S., Stashenko, E.E. 2010. Bioactivity against Tribolium castaneum Herbst (Coleoptera: Tenebrionidae) of Cymbopogon citratus and Eucalyptus citriodora essential oils grown in Colombia. Pest Management Science 66: 664-668.

Ootani, M.A., Aguiar, R.W. de S., Mello, A.V. de, Didonet, J., Portella, A.C.F., Nascimento, I.R. do. 2011. Toxicidade de óleos essenciais de eucalipto e citronela sobre Sitophilus zeamais MOTSCHULSKY (Coleoptera: Curculionidae). Bioscience Journal 27: 609-618.

Peixoto, M. F., Barbosa, R.V., Oliveira, R.R. da C., Fernandes, P.M., Costa, R.B da. 2009. Amostragem do ácaro rajado Tetranychus urticae Koch (Acari: Tetranychidae) e eficiência de acaricidas no seu controle na cultura do algodoeiro irrigado. Bioscience Journal 25: 24-32.

Pontes, W.J.T., Oliveira, J.C.S de, Câmara, C.A.G da, Gondim Júnior, M.G.C., Oliveira, J.V. de; Schwartz, M.O.E. 2007a. Atividade acaricida dos óleos essencias de folhas e frutos de Xylopia sericea sobre o ácaro rajado (Tetranychus urticae Koch). Química Nova 30: 838-841.

Pontes, W.J.T., Oliveira, J.C.S de, Câmara, C.A.G da, Lopes, A.C.H.R., Gondim Júnior, M.G.C., Oliveira, J.V. de, Barros, R., Schwartz, M.O.E. 2007b. Chemical composition and acaricidal activity of the leaf and fruit essential oils of Protium heptaphyllum (Aubl.) Marchand (Burseraceae). Acta Amazonica 37: 103-110. 
Robertson, J.L., Russell, R.M., Preisler, H.K., Savin, N.E. 2007. Bioassays with Arthropods. 2 ed. CRC Press, Boca Raton, USA. 199p.

Roh, H.S., Lee, B.H, Park, C.G. 2013. Acaricidal and repellent effects of myrtacean essential oils and their major constituents against Tetranychus urticae (Tetranychidae). Journal of Asia-Pacific Entomology 16: 245-249.

Sato, M.E., Silva, M.Z. da, Silva, R.B. da, Souza Filho M.F., Raga, A. 2009. Monitoramento da resistência de Tetranychus urticae Koch (Acari: Tetranychidae) a abamectin e fenpyroximate em diversas culturas no estado de São Paulo. Arquivos do Instituto Biológico 76: 217-223.

Souza, L.P., Zago, H.B., Costa, A.V., Stinguel, P., Valbon, W.R. 2015. Composição química e atividade acaricida do óleo essencial de ervade-santa-maria sobre o ácaro-rajado. Revista Caatinga 28: 160-166.

Viegas Júnior, C. 2003. Terpenos com atividade inseticida: uma alternativa para o controle químico de insetos. Química Nova 26:390- 400.

Vitti, A.M.S., Brito, J.O. 2003. Óleo essencial de Eucalipto. IPEF, Instituto de Pesquisas e Estudos Florestais. Documentos Florestais 17: 2-25.

Yaghoobi-Ershadi, M.R., Akhavan, A.A., Jahanifard, E., Vantandoost, H., Amin, G.H., Moosavi, L., Ramazani, A.R.Z., Abdoli, H., Arandian, M.H. 2006. Repellency effect of Myrtle essential oil and DEET against Phlebotomus papatasi, under laboratory conditions. Iranian Journal Public Health 35: 7-13.

Zandi-Sohani, N., Ramezani, L. 2015. Evaluation of five essential oils as botanical acaricides against the strawberry spider mite Tetranychus turkestani Ugarov and Nikolskii. International Biodeterioration \& Biodegradation 98: 101-106. 